\title{
The Cut-off Limits of Growth Hormone Response to the Insulin Tolerance Test Related to Body Mass Index for the Diagnosis of Adult Growth Hormone Deficiency
}

\author{
Valentina Gasco Alice Ferrero Alessandro Bisceglia Nunzia Prencipe \\ Valeria Cambria Fabio Bioletto Ezio Ghigo Mauro Maccario Silvia Grottoli
}

Department of Medical Science, Division of Endocrinology, Diabetes and Metabolism, University of Turin, Turin, Italy

\section{Keywords}

Insulin tolerance test · Growth hormone · GH deficiency ·

Sensitivity $\cdot$ Specificity

\begin{abstract}
Introduction: The diagnosis of growth hormone deficiency (GHD) in adults is based on a reduced GH response to provocative tests, such as the insulin tolerance test (ITT) and the $\mathrm{GH}$-releasing hormone $(\mathrm{GHRH})+$ arginine (ARG) test. However, the cut-off limits of peak $\mathrm{GH}$ response in lean subjects are not reliable in obese patients; this is noteworthy since adult GHD is often associated with obesity. To date, there are no ITT cut-offs related to body mass index (BMI). Objective: We aimed to evaluate the diagnostic cut-offs of $\mathrm{GH}$ response to the ITT in the function of BMI. Methods: The GH response to the ITT was studied in 106 patients with a history of hypothalamic-pituitary disease, a mean age of $48.2 \pm 12.4$ years, and a mean BMI of $26.8 \pm 6.1 \mathrm{~kg} / \mathrm{m}^{2}$ ). Patients were divided into lean, overweight, and obese groups according to their BMI. The lack of GH response to GHRH + ARG test was considered the gold standard for the diagnosis of GHD. The best $\mathrm{GH}$ cut-off in the ITT, defined as the one with the best sensitivity (SE) and specificity (SP), was identified using receiver-
\end{abstract}

operating characteristics curve (ROC) analysis. Results: The best $\mathrm{GH}$ cut-off in the ITT was $3.5 \mu \mathrm{g} / \mathrm{L}$ in lean subjects (SE 82.1\%; SP 85.7\%), $1.3 \mu \mathrm{g} / \mathrm{L}$ in overweight subjects (SE 74.1\%; SP 85.7\%), and $2.2 \mu \mathrm{g} / \mathrm{L}$ in obese subjects (SE 90.0\%; SP $50.0 \%)$. The diagnostic accuracy was $97.2,76.5$, and $76.7 \%$, respectively. Conclusions: Our data show that the ITT represents a reliable diagnostic tool for the diagnosis of adult GHD in lean subjects if an appropriate cut-off limit is assumed. Overweight and obesity strongly reduce the $\mathrm{GH}$ response to the ITT, GH BMI-related cut-off limits, and the diagnostic reliability of the test.

๑) 2020 S. Karger AG, Basel

\section{Introduction}

Adult growth hormone deficiency (GHD) is a heterogeneous disorder that may occur during childhood or adult life, resulting from a variety of causes including structural lesions, genetic abnormalities, trauma, infiltrative diseases, and surgery or irradiation to the pituitary gland and/or hypothalamus [1]. This condition is characterized by altered body composition, glucose intolerance, abnormal lipid profile, premature atherosclerosis, osteo- karger@karger.com

(C) 2020 S. Karger AG, Basel

www.karger.com/nen

Karger!
Valentina Gasco

Divisione di Endocrinologia, Diabetologia e Metabolismo

Dipartimento di Scienze Mediche, ASOU “Città della Salute e Della Scienza” di Torino

C.so Dogliotti 14, IT-10126 Turin (Italy)

valentina.gasco@unito.it 
porosis, an impaired quality of life, and increased mortality [2-4]. Treatment with GH replacement improves most of these abnormalities $[5,6]$. However, due to the high cost of GH replacement [7] and the concerns about potential long-term risks, particularly the development of diabetes mellitus or malignancy, it is imperative that the correct diagnosis is established so that appropriate $\mathrm{GH}$ replacement is offered to adults who are truly GH-deficient, and not just for nonmedical conditions such as aging or sports.

Adult GHD diagnosis depends on the demonstration of a subnormal rise in peak serum $\mathrm{GH}$ level in response to $\geq 1 \mathrm{GH}$ stimulation tests $[1,8,9]$. All GH stimulation tests are based on the concept that a pharmacological agent stimulates pituitary GH secretion, with peak GH levels detectable by timed frequent serum sampling after administration of the stimulus.

One of the caveats in interpreting the results of most GH stimulation tests in adults is the high prevalence of obesity in the general population, including patients with acquired hypothalamic-pituitary disease [10]. It must be remembered that obesity is a state of functional, relative GHD, with decreased spontaneous secretion, pulses, and half-life of GH [11-13]. In addition, decreased GH responsiveness to all stimulation tests has been demonstrated in subjects with obesity and/or abdominal adiposity [14-16].

The insulin tolerance test (ITT) is the test of reference for the diagnosis of adult GHD. At the same time, GHreleasing hormone $(\mathrm{GHRH})$, in combination with arginine (ARG) or GH-releasing peptide-6 (GHRP-6), is recognized as being equally reliable $[1,8,9]$. Glucagon stimulation is also considered to be of good diagnostic value [17]. The ITT has traditionally been accepted as the gold standard test for the assessment of adult GHD, with a GH cut-off of 3-5 $\mu \mathrm{g} / \mathrm{L}$ during adequate hypoglycemia (blood glucose $<40 \mathrm{mg} / \mathrm{dL}$ ); however, to date, the test has no body mass index (BMI)-related cut-off for the diagnosis of adult GHD.

The alternative tests, i.e., GHRH + ARG, GHRH + GHRP-6, and glucagon stimulation, even though they present well-defined cut-off limits and normative BMIbased reference values [18-20], are either not available in some countries (due to discontinued production of the GHRH analog by EMD Serono in 2008) or have substantial limitations, including requiring intramuscular administration, being of long duration, and/or having low accuracy.

The aim of this study was to define the diagnostic value of the ITT and evaluate the diagnostic cut-off limits of peak GH response to the test in lean, overweight, and obese individuals. For this purpose, we studied the GH response to the ITT in patients with a history of pituitary disease. The lack of GH response to GHRH + ARG using BMI-related cut-offs was considered as the diagnostic gold standard.

\section{Materials and Methods}

We studied 164 patients with a history of pituitary disease who were referred to the Neuroendocrinology Clinic of our center from 1 January 2017 to 1 January 2019 for the evaluation of GH secretion.

We studied the GH response to the ITT (0.1-0.15 IU/kg regular insulin injected i.v. at $0 \mathrm{~min}$ in normal-weight and overweight/ obese subjects, respectively; sampling every 15 min from 0 to +90 min). Insulin growth factor I (IGF-I) and glucose levels were recorded during the ITT in all patients. Patients were also tested with GHRH $(1 \mu \mathrm{g} / \mathrm{kg}$ i.v. at $0 \mathrm{~min})+\mathrm{ARG}(0.5 \mathrm{~g} / \mathrm{kg}$ i.v. from 0 to +30 $\mathrm{min}$ ). Sampling was performed every $15 \mathrm{~min}$ from +30 to $+60 \mathrm{~min}$.

All subjects underwent the 2 testing sessions in a random order and at least 3 days apart. After an overnight fast, each test was conducted in the morning (8.00-8.30 a.m.), 30 min after an indwelling catheter had been placed into a forearm vein that was kept patent by slow infusion of isotonic saline.

Forty-one patients were excluded because either the ITT ( $n=$ $28)$ or the GHRH + ARG ( $n=13)$ were missing, 14 were excluded due to lacking a correct hypoglycemia (glucose $\leq 40 \mathrm{mg} / \mathrm{dL}$ ) during the ITT, and 3 patients did not give their informed consent. The final study population therefore consisted of 106 patients (61 males and 45 females; mean age $48.2 \pm 12.4$ years; mean BMI 26.8 \pm 6.1 ). Their clinical characteristics are reported in Table 1 .

Serum GH levels were measured in duplicate by the IRMA method (IRMA GH, Beckman Coulter, Czech Republic). The IRMA assay of GH is a sandwich-type assay. The kit utilizes mouse monoclonal antibodies directed against 2 different epitopes of the molecule. The antibodies recognize the $22-\mathrm{kDa}$ monomer, the dimer, and the GH bound to its binding protein. The calibrators are calibrated against the international standard WHO 2nd IS 98/574 in human serum. The sensitivity of the assay was $0.033 \mu \mathrm{g} / \mathrm{L}$. The inter- and intra-assay coefficients of variation (CV) were 9.0$14.0 \%$ and $2.4-6.5 \%$, respectively.

Serum IGF-I levels were measured in duplicate by the RIA method (SM-C-RIA-CT, DIAsource ImmunoAssays, Belgium) after extracting acid-ethanol to avoid interference by binding proteins. The sensitivity of the method was $0.25 \mu \mathrm{g} / \mathrm{L}$. The inter- and intra-assay CV were $6.8-14.9 \%$ and $4.5-7.0 \%$, respectively. IGF-I levels are expressed both as an absolute value and as a standard deviation score (SDS) of the mean normal value. The SDS for each subject was calculated in accordance with the published normality data on a population of 547 healthy Italian subjects [21].

Plasma glucose levels $(\mathrm{mg} / \mathrm{dL})$ were measured by gluco-oxidase colorimetric method (GLUCOFIX, Menarini Diagnostici, Florence, Italy). All samples from an individual subject were analyzed together. The results are expressed as mean \pm SD

Statistical analysis was performed using the Friedman ANOVA, Kendall concordance test and Wilcoxon matched-pairs test to 
Table 1. Clinical features of the 106 patients with a history of hypothalamicpituitary disease

\begin{tabular}{lc}
\hline Male/female & $61 / 45(57.5 / 42.5)$ \\
Age, years & $48.2 \pm 12.4$ \\
BMI, kg/m ${ }^{2}$ & $26.8 \pm 6.1$ \\
$\quad$ Lean subjects & $42(39.6)$ \\
Overweight subjects & $34(32.1)$ \\
Obese subjects & $30(28.3)$ \\
Subjects with/without other pituitary deficits (except GHD), $n$ & $65 / 41$ \\
Pathology & $106(100)$ \\
Pituitary tumors & $81(76.4)$ \\
Primary + secondary (due to Sheehan syndrome) empty sella & $10+1(10.4)$ \\
Idiopathic & $7(6.6)$ \\
Pituitary hypoplasia & $6(5.7)$ \\
Traumatic brain injury & $1(0.9)$ \\
Treatment of the pituitary tumors & $81(100)$ \\
Neurosurgery & $53(65.4)$ \\
Neurosurgery + radiotherapy & $17(21.0)$ \\
None & $11(13.6)$
\end{tabular}

Values are expressed as $n(\%)$ or mean $\pm \mathrm{SD}$, unless otherwise indicated. compare multiple and 2 dependent samples, respectively, while the Mann-Whitney U test was applied to compare 2 independent samples. The correlation between variables was sought by calculating the Spearman coefficient.

The lack of GH response to GHRH + ARG was assumed as the gold standard for the diagnosis of GHD. Thus, as per the GHD diagnosis guideline [1], GHD was defined by using the BMI cut-offs (i.e., a GH peak of $\leq 11.0, \leq 8.0$, and $\leq 4.0 \mu \mathrm{g} / \mathrm{L}$ in lean, overweight, and obese subjects, respectively) [20].

We tried to identify the best GH cut-off in the ITT for the diagnosis of GHD using receiver-operating characteristics curve (ROC) analysis. The best cut-off in a ROC curve is the closest point to that with the theoretical maximum of both sensitivity (SE) and specificity (SP). The area under the ROC curve (ROC AUC) represents the probability of correctly distinguishing between affected and nonaffected individuals. The perfect diagnostic test has a ROC curve that passes through the upper left-hand corner (AUC 1), being the true-positive fraction 1.0 or $100 \%$ (perfect SE), and the false-positive fraction 0 (perfect SP). Tests with an AUC $<0.5$ do not discriminate between affected and nonaffected subjects [22].

The diagnostic cut-off points were calculated for the lean (BMI $<25 \mathrm{~kg} / \mathrm{m}^{2}$ ), overweight (BMI $25-29.9 \mathrm{~kg} / \mathrm{m}^{2}$ ), and obese (BMI $\geq 30 \mathrm{~kg} / \mathrm{m}^{2}$ ) groups. Subsequently, we unified the overweight and obese patients into a single group and calculated a single cut-off for this new group.

For each identified cut-off, we also calculated the positive predictive value (PPV), negative predictive value (NPV), positive likelihood ratio (LHR+), and negative likelihood ratio (LHR-). LHR+ $>1$ indicates that a positive result to the test is associated with the disease; LHR $-<1$ indicates that a negative result is associated with absence of the disease. Tests where the LHR lies close to 1 have little practical significance, as the posttest probability (odds) is little different from the pretest probability and, as such, is used primarily for diagnostic but not for screening purposes. When the LHR+ is $>5$ or the LHR- is $<0.2$ (i.e., 1/5), these can be applied to the pretest probability of a patient having the disease tested for estimating a posttest probability of the disease state existing [23].
The accuracy of the test was defined as the probability of a person who has the disease and one who does not have the disease testing positive and negative, respectively.

\section{Results}

Based on GH response to GHRH + ARG, 75 patients were distinguished as having GHD (44 males and $31 \mathrm{fe}-$ males) and 31 as having no GHD (noGHD: 17 males and 14 females). These 2 groups differed for age ( $50.3 \pm 10.5$ and $43.6 \pm 14.8$ years, respectively; $p<0.05$ ) but not for BMI $\left(27.1 \pm 6.0\right.$ and $26.0 \pm 6.3 \mathrm{~kg} / \mathrm{m}^{2}$, respectively; $\left.p=0.45\right)$.

IGF-I levels in the GHD group were lower than in the noGHD group when expressed both as mean and SDS levels $(105.6 \pm 56.2$ vs. $179.8 \pm 91.6 \mu \mathrm{g} / \mathrm{L}, p<0.0001 ;-1.0$ \pm 0.8 vs. $-0.7 \pm 1.0, p<0.01$ ); the 2 groups showed a clear overlap (IGF-I range: $20-317 \mu \mathrm{g} / \mathrm{L}$ [GHD] and 76-512 $\mu \mathrm{g} / \mathrm{L}[\mathrm{noGHD}])$.

The mean GH response to the ITT in the GHD group was lower than that in the noGHD group ( $1.4 \pm 1.7$ vs. 7.3 $\pm 8.8 \mu \mathrm{g} / \mathrm{L} ; p<0.0001)$. Similarly, the mean GH response to GHRH + ARG in the GHD group was lower than in the noGHD group $(2.8 \pm 2.3$ vs. $19.9 \pm 17.2 \mu \mathrm{g} / \mathrm{L} ; p<0.0001)$ (Fig. 1).

The GH response to the ITT in the GHD group (1.4 \pm $1.7 \mu \mathrm{g} / \mathrm{L})$ was lower than that during GHRH + ARG (2.8 $\pm 2.3 \mu \mathrm{g} / \mathrm{L})(p<0.0001 ;$ Fig. 1$)$. Similarly, the GH response to the ITT in the noGHD group $(7.3 \pm 8.8 \mu \mathrm{g} / \mathrm{L})$ was lower than that during GHRH + ARG $(19.9 \pm 17.2 \mu \mathrm{g} / \mathrm{L})(p<$ 0.0001; Fig. 1). 
Fig. 1. Peak GH response (mean $\pm \mathrm{SD}$ ) to ITT and GHRH + ARG test in patients with GHD and without GHD (noGHD) as a function of group (a) and the type of test (b); ${ }^{*} p<0.0001$.

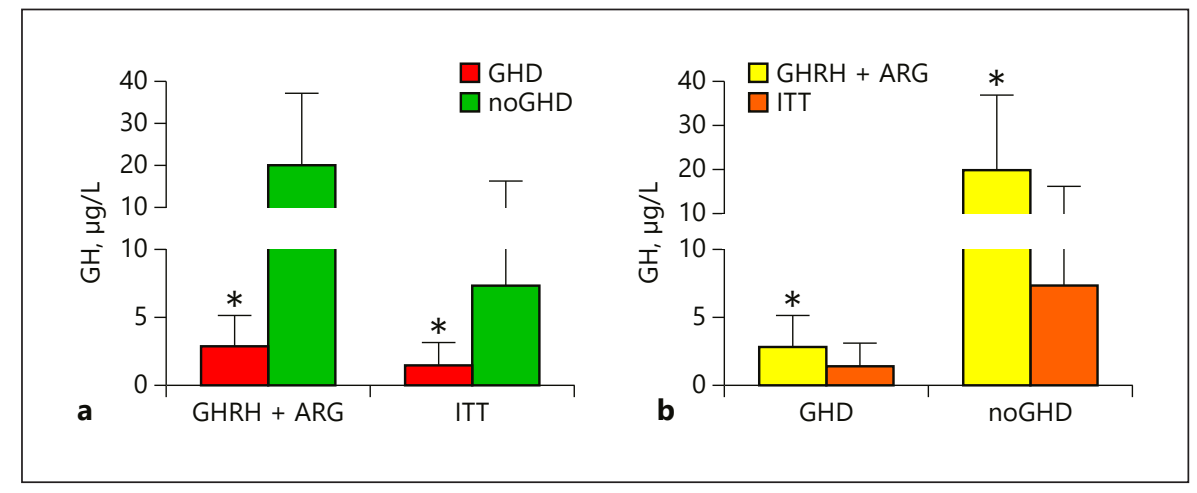

Fig. 2. Mean GH (mean \pm SD) curve to ITT in patients with GHD and without GHD (noGHD) (a) and mean glucose (mean \pm SD) curve to ITT in GHD and noGHD (b). ${ }^{*} p<0.05$ vs. basal; ${ }^{* *} p<0.0001$ vs. basal; ${ }^{\circ} p<0.0005$ vs. basal; ${ }^{\$ \S} p<0.00005$ vs. basal; ${ }^{\S} p<0.05$ GHD vs. noGHD.

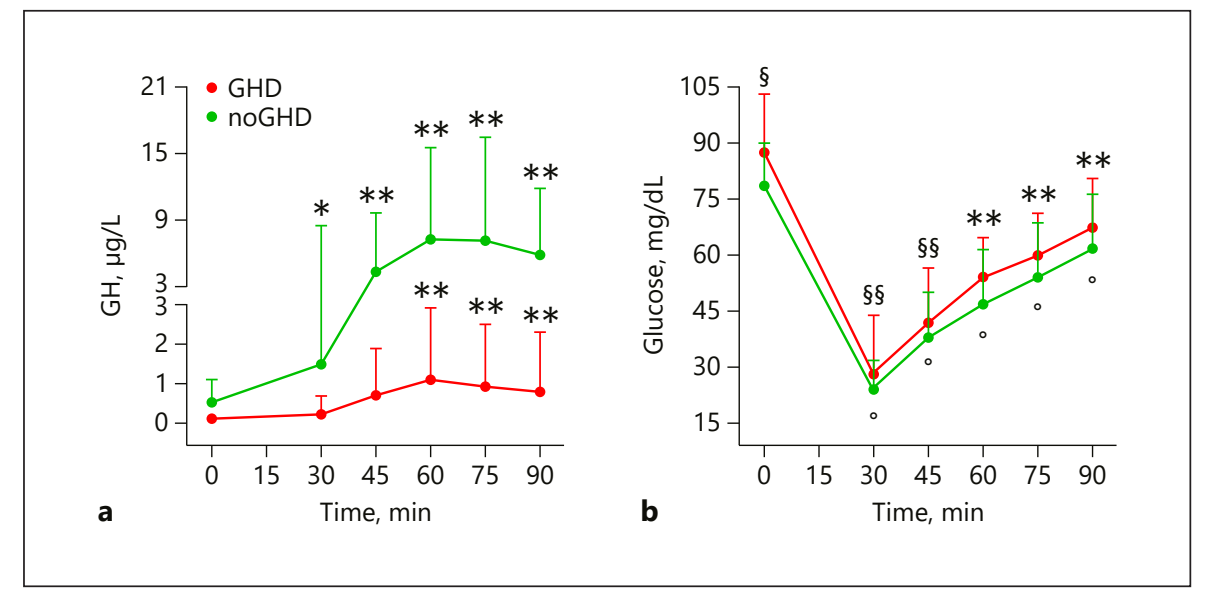

The individual peak GH response to the ITT occurred in all subjects between +45 and +90 min with a curve $\mathrm{C}_{\max }$ at +60 min in both the GHD and noGHD groups (Fig. 2a).

The GH response to both the GHRH + ARG and the ITT in the whole population was not associated with age $(\mathrm{GHRH}+$ ARG: $r=-0.18, p=0.06$; ITT: $r=-0.10, p=$ $0.29)$ and the absence of significance was also confirmed in the GHD group (GHRH + ARG: $r=0.0, p=1.0$; ITT: $r=0.10, p=0.90)$ and in the noGHD group (GHRH + ARG: $r=-0.21, p=0.26$; ITT: $r=-0.04, p=0.84$ ).

The GH response to GHRH + ARG in the whole population was negatively associated with BMI $(r=-0.28$, $p<0.005)$, and a negative correlation was confirmed in both the GHD group $(r=-0.28, p<0.05)$ and the noGHD group $(r=-0.61, p<0.001)$. The GH response to the ITT in the whole population was negatively associated with BMI $(r=-0.22, p<0.05)$, and a negative correlation was confirmed in the noGHD group $(r=-0.45, p<0.05)$ but not in the GHD group $(r=-0.10, p=0.42)$.

Basal glucose levels were higher in the GHD group $(87.4 \pm 15.4 \mathrm{mg} / \mathrm{dL})$ than in the noGHD group $(78.4 \pm$

GH Response to ITT Related to BMI
$11.5 \mathrm{mg} / \mathrm{dL}, p<0.05)$; however, there was no difference between glucose nadir during the ITT in the GHD and noGHD groups $(24.8 \pm 7.8$ vs. $23.7 \pm 7.8 \mathrm{mg} / \mathrm{dL} ; p=0.73)$ (Fig. 2b).

The best GH cut-offs in the ITT were: (i) $3.5 \mu \mathrm{g} / \mathrm{L}$ in lean subjects with SE and SP of 82.1 and $85.7 \%$, respectively; (ii) $1.3 \mu \mathrm{g} / \mathrm{L}$ in overweight subjects with SE and SP of 74.1 and $85.7 \%$, respectively; (iii) $2.2 \mu \mathrm{g} / \mathrm{L}$ in obese subjects with SE and SP of 90.0 and $50.0 \%$, respectively; (iv) $1.3 \mu \mathrm{g} / \mathrm{L}$ in overweight and obese subjects with SE and SP of 72.3 and $70.6 \%$, respectively (Fig. 3).

The PPV, NPV, ROC AUC, LHR+, LHR-, and diagnostic accuracy of these cut-off limits in the ITT are reported in Table 2. Thus, in the case of a positive ITT result, the prediction of GHD was good in the lean and overweight subjects (LHR+ 5.8 and 5.2, respectively), and poor in the obese subjects $(\mathrm{LHR}+1.8)$. Likewise, in the case of a negative result, the prediction of the absence of disease was good in the lean and obese subjects (LHR- 0.2 for both) but insufficient in the overweight subjects (LHR- 0.3). The identified cut-off unifying the over- 


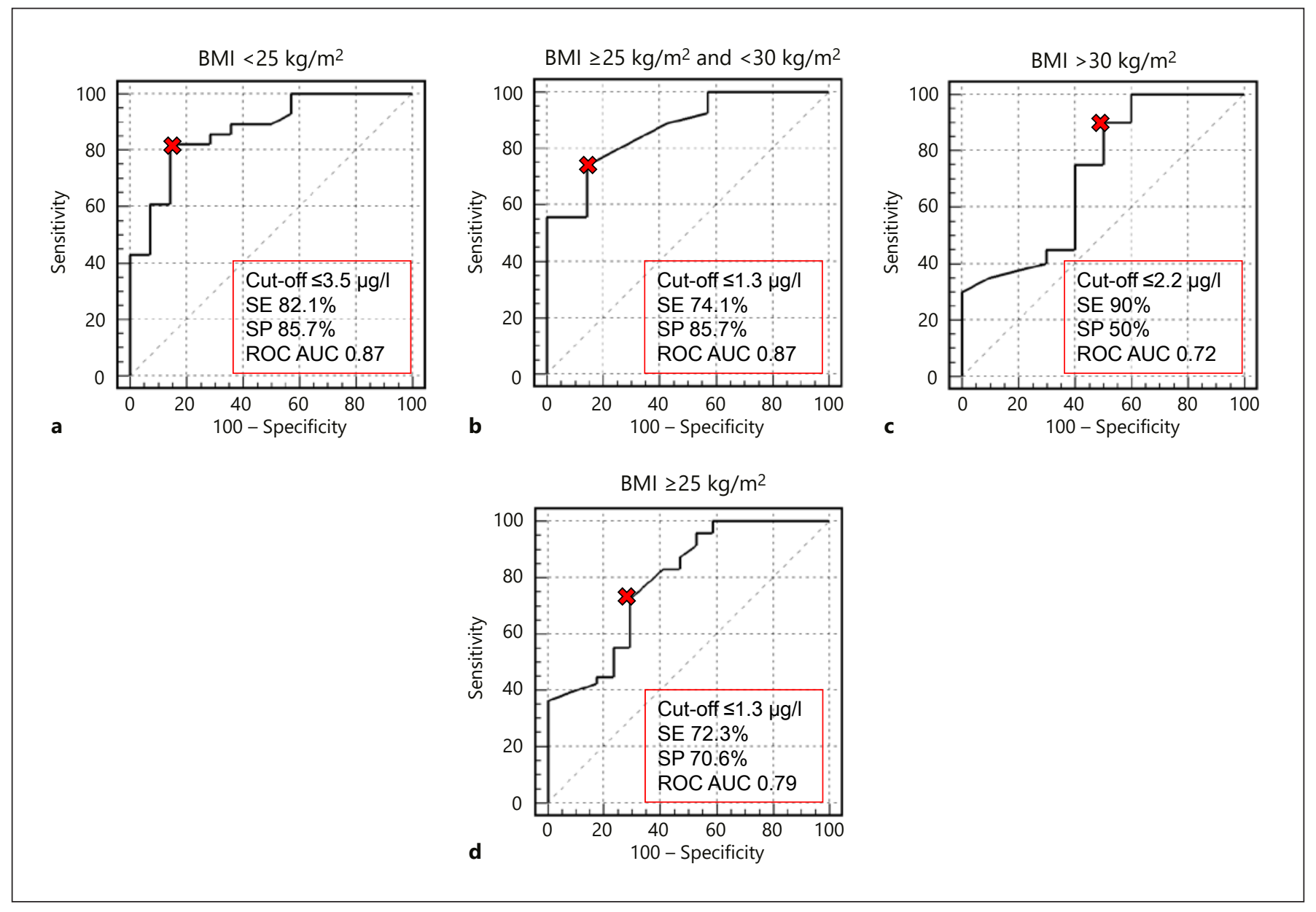

Fig. 3. ROC analysis to identify the best GH cut-off for the ITT in lean (a), overweight (b), obese (c), and overweight and obese (d) patients. SE, sensitivity; SP, specificity; ROC AUC, area under the ROC curve.

weight and obese patient groups was characterized by insufficient LHR+ and LHR- values (Table 2).

\section{Side Effects}

Administration of GHRH + ARG did not cause any relevant side effects except for face flushing observed in 28 patients. As expected, all subjects developed typical signs and symptoms of hypoglycemia during the ITT; it was necessary to administer a glucose solution i.v. for evidence of prolonged hypoglycemia in 10 patients.

\section{Discussion}

To our knowledge, this is the first study that identifies the cut-off limits of the GH response to the ITT related to BMI for the diagnosis of adult GHD. Our results, based on a large group of patients with a history of hypothalamic-pituitary disease, confirmed that the ITT is a reliable provocative test for the diagnosis of severe GHD in adults, at least in normal-weight subjects. Our data specifically showed that:

1. (a) The ITT is basically less potent than the GHRH + ARG test in noGHD but also in GHD

2. (b) As expected, as well as for GHRH + ARG, the GH response to the ITT is more reduced in GHD than in noGHD

3. (c) As with the other tests, GH response to the ITT is negatively associated with BMI, at least in the whole population and in noGHD

4. (d) As demonstrated by the ROC curve, when BMI adjusted cut-off limits are applied, the ITT presents a good SE and SP (it was able to recognize $81.3 \%$ of the patients with severe GHD and $74.2 \%$ of patients without GHD) 
Table 2. GH cut-off limits in the ITT identified with ROC analysis in lean, overweight, and obese patients

\begin{tabular}{|c|c|c|c|c|c|c|c|c|}
\hline GH level & $\mathrm{SE}, \%$ & $\mathrm{SP}, \%$ & PPV, \% & NPV, \% & $\mathrm{ROC} A U C \pm S D$ & LHR+ & LHR- & Diagnostic accuracy, \% \\
\hline$<3.5 \mu \mathrm{g} / \mathrm{L}$ in lean subjects & 82.1 & 85.7 & 92.0 & 70.6 & $0.87 \pm 0.06$ & 5.8 & 0.2 & 97.2 \\
\hline$<1.3 \mu \mathrm{g} / \mathrm{L}$ in overweight subjects & 74.1 & 85.7 & 95.2 & 46.2 & $0.87 \pm 0.09$ & 5.2 & 0.3 & 76.5 \\
\hline$<1.3 \mu \mathrm{g} / \mathrm{L}$ in overweight + obese subjects & 72.3 & 70.6 & 87.2 & 48.0 & $0.79 \pm 0.07$ & 2.5 & 0.4 & 71.9 \\
\hline
\end{tabular}

SE, sensitivity; SP, specificity; PPV, positive predictive value; NPV, negative predictive value; ROC AUC, area under the ROC curve; LHR+, positive likelihood ratio; LHR-, negative likelihood ratio.

5. (e) Overweight and obesity strongly reduce the GH response to the ITT, its weight-related cut-off limit, and its diagnostic reliability.

GHD is a heterogeneous disorder that may occur during childhood or adulthood, resulting from a variety of causes [1]. The condition is characterized by altered body composition, glucose intolerance, an abnormal lipid profile, premature atherosclerosis, osteoporosis, an impaired quality of life, and increased mortality [2-4]. Treatment with GH replacement improves most of these abnormalities $[5,6]$. However, due to the high cost of GH replacement and concerns about the potential long-term risks, particularly the development of diabetes mellitus or malignancy, it is imperative that the correct diagnosis is established so that appropriate GH replacement is offered to adults who are truly GH-deficient, and not just for nonmedical conditions such as aging or sports.

Adult GHD diagnosis depends on the demonstration of a subnormal rise of $\mathrm{GH}$ level in response to $\geq 1 \mathrm{GH}$ stimulation tests $[1,8,9]$. All GH stimulation tests are based on the concept that a pharmacological agent stimulates pituitary GH secretion, with peak GH levels detectable by timed frequent serum sampling after administration of the stimulus.

The fact that the GH response to the ITT in noGHD and GHD subjects was clearly lower than that induced by GHRH + ARG is in full agreement with other studies that have shown the very powerful GH-stimulating effect of GHRH + ARG [24-27]. On the other hand, the fact that the GH response to the ITT in noGHD subjects was lower than that induced by GHRH + ARG could at least partially derive from the methods used in this study to define patients as having no GHD. Patients who showed a sufficient response to GHRH + ARG were considered to not be affected by a severe somatotropic deficit; however, it has been pointed out that a normal response to GHRH + ARG does not exclude a GHD with hypothalamic pathogenesis, which can, instead, be identified by the ITT [28].
It seems unlikely that the difference in age observed in the GHD and noGHD groups could have influenced the GH response to the ITT, since it has already been shown in adults that the response of GH to the ITT does not depend on age [25]. As confirmation of this, in our work there was no significant correlation between age and GH peak to the ITT, in the whole population or in the GHD and noGHD subgroups.

On the other hand, our work also highlights the wellknown negative association between the GH response to stimulation tests and BMI, also when the test concerned is the ITT. The negative influence of adiposity on the spontaneous and stimulated secretion of GH was already clearly demonstrated many years ago [29-34]. The impairment of GH secretion in the case of obesity could be due to both neuroendocrine (the hypoactivity of neurons secreting GHRH and/or somatostatinergic hyperactivity) and metabolic (chronically elevated free fatty acid levels and hyperinsulinism) changes [29-34]. Regardless of the pathophysiological explanation for somatotropic insufficiency in obesity, the clear and progressive reduction in the GH response to any stimulation test has clinical implications when these tests are used to demonstrate a GHD [33]. Indeed, one of the caveats in interpreting the results of all the GH stimulation tests in adults is the high prevalence of obesity in the general population, including patients with hypothalamic-pituitary disease [10]. Even if the ITT has traditionally been accepted as the gold standard for the assessment of adult GHD, to date, the test has no cut-off BMI related to the diagnosis of adult GHD. With this in mind, the first objective of this study was therefore to identify the cut-off limits related to BMI of the GH response to the ITT, to allow the correct identification of patients with severe GHD, even under conditions of overweight and obesity that often complicate the condition of GHD itself. Furthermore, the feasibility of a test active at both the hypothalamic and pituitary levels would be more advantageous than a stimulation test like 
the GHRH + ARG, which is extremely powerful and endowed with excellent specificity, but acts mainly at the pituitary level and is thus associated with the risk of a false-negative response in patients with GHD due to hypothalamic damage [1, 28, 35-37]. Finally, it should be emphasized that in some situations (such as currently in the USA), the GHRH + ARG test, although maximal and equipped with specific BMI-dependent cut-off limits [20], does not find application as a consequence of the withdrawal of the GHRH peptide from the market, which is currently still available in Europe in the form of GHRH $1-44$.

As demonstrated by the ROC curves, the cut-off limits proposed for the ITT are characterized by a good SE and SP, at least in lean patients, being able to identify $23 / 28$ (82.1\%) patients with severe GHD and 12/14 (85.7\%) patients without GHD.

The usefulness of the ITT in lean subjects is also supported by the good LHR+ $(>5)$ and LHR- $(<0.2)$ of the identified cut-off [23]. Furthermore, it must be noted that the ROC AUC for the cut-off in lean subjects is close to 1 , indicating the excellent informative value of the test.

The cut-off identified in our study in overweight subjects, and even more so in obese subjects, was characterized by considerably reduced diagnostic accuracy of the test, compared to that for normal-weight subjects. For this reason, the ITT cannot represent the diagnostic tool of first choice in overweight and obese patients suspected of GHD; in these patients, GHRH + ARG remains the most suitable test. For this reason, and in view of the results obtained, we decided to unify the overweight and obese group, trying to enhance the specificity of the test, as we considered it was more important to be sure to exclude patients not affected by GHD rather than to not treat patients with GHD. However, with this new approach, the cut-off identified for the overweight/obese group was characterized by both an insufficient LHR+ and an insufficient LHR-.

Lastly, it must be emphasized that the cut-offs identified are valid with the assay used in this study, but that it is possible to carry out a comparison (Bland-Altman plot) with any assay to identify any correction factors. Accurate measurement of serum GH levels is critical for making the diagnosis of adult GHD. It is well known that specific GH cut-off levels in GH stimulation tests must be interpreted in the context of the analytical method used. The lack of GH assay standardization has led to major differences in the values of hormone concentrations obtained with different assays [38]. The foremost fundamental requirement for improving the compara- bility of GH assay methods is to use a single universally accepted standard for each hormone assay. Unfortunately, at the moment, this is not the case [38]. An important contributor to differences in GH assay is the use of different calibrator materials [39]. Currently, not all GH assays are calibrated to a common international reference preparation [38]. However, the assay used in our study was calibrated against the international standard WHO 2nd IS 98/574, as suggested in the Consensus Statement on the standardization and evaluation of GH and IGF assays [40].

It must be remembered that the cut-off of $3 \mu \mathrm{g} / \mathrm{L}$ currently used worldwide for the interpretation of the ITT derives from a study carried out in 1994 by Hoffman et al. [41] based on GH assessment performed with an old assay using an old standard. They evaluated GH response to the ITT in only 23 patients considered GH-deficient due to organic pituitary disease, and in 35 sex-matched normal subjects of a similar age and BMI. A study carried out in 2002 by Biller et al. [42], to evaluate the relative utility of 6 different methods of testing for adult GHD (including the ITT) and develop diagnostic cut-offs for each of these tests, was conducted on only 39 patients and 34 age-, sex-, estrogen status-, and BMI-matched control subjects. A third group of 21 patients with adult-onset hypothalamic-pituitary disease and no more than one additional pituitary hormone deficiency was also studied. The results of that particular study, although conducted on a small group, were incorporated into the guidelines of the Endocrine Society [1], which underline that an analysis of different cut-off limits of $\mathrm{GH}$ response to the ITT related to BMI to diagnose adult GHD has not yet been performed [1]. Our study is steered precisely in this direction and, unlike previous studies on this topic, we considered only patients with a known history of hypothalamic-pituitary disease and therefore having a risk of developing GHD, deliberately not considering normal subjects for comparison. It is a known fact that when a diagnostic study starts by including patients who have already received a diagnosis of the target condition and uses a group of healthy volunteers as the control group, it is likely that the sensitivity and specificity will both be higher than in a study made up of patients who are only suspected of having the target condition; in such studies, the results obtained cannot be applied to the clinical setting [43].

Another interesting aspect that emerged from this study was the observation of the times within which the maximum GH response to the ITT is recorded. In almost all cases, the peak in the test was in the period from +45 
min to +90 min after administering insulin. This allows us to foresee that, similar to what has been proposed for the GHRH + ARG test, for GHRH + GHRP-6 and GHRP2 tests $[26,44,45]$, the duration of the observation time after the stimulus can be usefully restricted to this period. Obviously, this would have a positive impact on both the patient and cost of hormonal determination and the time for which the medical staff are occupied.

Finally, the data from this study demonstrate that the measurement of total IGF-I levels shows a considerable overlap between GHD and noGHD, confirming that normal IGF-I levels do not exclude the existence of severe GHD in adults $[1,8,9]$.

In conclusion, our study suggests that the ITT is a reliable tool for the diagnosis of GHD in adults, and that it can be used instead of GHRH + ARG which was used until now to identify the condition of GHD $[1,8,9]$. However, attention must be paid to the confounding factors exerted by the condition of overweight and obesity on the interpretation of the $\mathrm{GH}$ response to this test. As previously reported, obesity reduces the response of GH to any stimulation test [29-34]. Consequently, appropriate threshold values related to BMI are mandatory to avoid false-positive diagnoses of severe GHD in obese adults. Indeed, the impact of obesity on the response of GH to the ITT seems particularly important.

\section{Acknowledgement}

The authors thank Mrs. M. Taliano for her skillful technical assistance.

\section{Statement of Ethics}

The protocol was in accordance with the principles of the Declaration of Helsinki and was approved by the local ethics committee. Subjects gave their informed consent to participate in the study.

\section{Conflict of Interest Statement}

The authors have no conflicts of interest to declare.

\section{Funding Sources}

The study did not receive any funding.

\section{Author Contributions}

V.G., A.F., A.B., N.P., V.C., and F.B. collected the anamnestic as well as the biochemical data for each patient. V.G. performed the data analysis, the figures designing and the manuscript writing. E.G., M.M., and S.G. verified the analytical methods and supervised the manuscript drafting. All authors discussed the results and contributed to the final manuscript.

\section{References}

1 Molitch ME, Clemmons DR, Malozowski S, Merriam GR, Vance ML; Endocrine Society. Evaluation and treatment of adult growth hormone deficiency: an Endocrine Society clinical practice guideline. J Clin Endocrinol Metab. 2011 Jun;96(6):1587-609.

2 Burman P, Mattsson AF, Johannsson G, Höybye C, Holmer H, Dahlqvist P, et al. Deaths among adult patients with hypopituitarism: hypocortisolism during acute stress, and de novo malignant brain tumors contribute to an increased mortality. J Clin Endocrinol Metab. 2013 Apr;98(4):1466-75.

3 Simpson H, Savine R, Sönksen P, Bengtsson BA, Carlsson L, Christiansen JS, et al.; GRS Council. Growth hormone replacement therapy for adults: into the new millennium. Growth Horm IGF Res. 2002 Feb;12(1):133.

4 Svensson J, Bengtsson BA, Rosén T, Odén A, Johannsson G. Malignant disease and cardiovascular morbidity in hypopituitary adults with or without growth hormone replacement therapy. J Clin Endocrinol Metab. 2004 Jul;89(7):3306-12.

5 Jørgensen AP, Fougner KJ, Ueland T, Gudmundsen O, Burman P, Schreiner T, et al. Fa- vorable long-term effects of growth hormone replacement therapy on quality of life, bone metabolism, body composition and lipid levels in patients with adult-onset growth hormone deficiency. Growth Horm IGF Res. 2011 Apr;21(2):69-75.

6 Widdowson WM, Gibney J. The effect of growth hormone replacement on exercise capacity in patients with GH deficiency: a metaanalysis. J Clin Endocrinol Metab. 2008 Nov; 93(11):4413-7.

7 Vance ML. Can growth hormone prevent aging? N Engl J Med. 2003 Feb;348(9):779-80.

8 Cook DM, Yuen KC, Biller BM, Kemp SF, Vance ML; American Association of Clinical Endocrinologists. American Association of Clinical Endocrinologists medical guidelines for clinical practice for growth hormone use in growth hormone-deficient adults and transition patients - 2009 update. Endocr Pract. 2009 Sep-Oct; 15 Suppl 2:1-29.

9 Ho KK; 2007 GH Deficiency Consensus Workshop Participants. Consensus guidelines for the diagnosis and treatment of adults with GH deficiency II: a statement of the GH Research Society in association with the European Society for Pediatric Endocrinology,
Lawson Wilkins Society, European Society of Endocrinology, Japan Endocrine Society, and Endocrine Society of Australia. J Eur J Endocrinol. 2007 Dec;157(6):695-700.

10 Verhelst J, Abs R. Cardiovascular risk factors in hypopituitary GH-deficient adults. Eur J Endocrinol. 2009 Nov;161 Suppl 1:S41-9.

11 Iranmanesh A, Lizarralde G, Veldhuis JD. Age and relative adiposity are specific negative determinants of the frequency and amplitude of growth hormone $(\mathrm{GH})$ secretory bursts and the half-life of endogenous GH in healthy men. J Clin Endocrinol Metab. 1991 Nov;73(5):1081-8.

12 Van Dam EW, Roelfsema F, Helmerhorst FH, Frölich M, Meinders AE, Veldhuis JD, et al. Low amplitude and disorderly spontaneous growth hormone release in obese women with or without polycystic ovary syndrome. J Clin Endocrinol Metab. 2002 Sep;87(9): 4225-30.

13 Veldhuis JD, Iranmanesh A, Ho KK, Waters MJ, Johnson ML, Lizarralde G. Dual defects in pulsatile growth hormone secretion and clearance subserve the hyposomatotropism of obesity in man. J Clin Endocrinol Metab. 1991 Jan;72(1):51-9. 
14 Vizner B, Reiner Z, Sekso M. Effect of 1-dopa on growth hormone, glucose, insulin, and cortisol response in obese subjects. Exp Clin Endocrinol. 1983 Jan;81(1):41-8.

15 Cordido F, Dieguez C, Casanueva FF. Effect of central cholinergic neurotransmission enhancement by pyridostigmine on the growth hormone secretion elicited by clonidine, arginine, or hypoglycemia in normal and obese subjects. J Clin Endocrinol Metab. 1990 May; 70(5):1361-70.

16 Hartman M. Physiological regulators of growth hormone secretion. In: Juul A, Jørgensen JOL, editors. Growth Hormone in Adults, 2nd ed. Cambridge: Cambridge University Press; 2000. pp. 3-53.

17 Gordon MB, Levy RA, Gut R, Germak J. Trends in growth hormone stimulation testing and growth hormone dosing in adult growth hormone deficiency patients: results from the ANSWER program. Endocr Pract. 2016 Apr;22(4):396-405.

18 Kelestimur F, Popovic V, Leal A, Van Dam PS, Torres E, Perez Mendez LF, et al. Effect of obesity and morbid obesity on the growth hormone $(\mathrm{GH})$ secretion elicited by the combined GHRH + GHRP-6 test. Clin Endocrinol (Oxf). 2006 Jun;64(6):667-71.

19 Dichtel LE, Yuen KC, Bredella MA, Gerweck AV, Russell BM, Riccio AD, et al. Overweight/ Obese adults with pituitary disorders require lower peak growth hormone cutoff values on glucagon stimulation testing to avoid overdiagnosis of growth hormone deficiency. J Clin Endocrinol Metab. 2014 Dec;99(12):4712-9.

20 Corneli G, Di Somma C, Baldelli R, Rovere S, Gasco V, Croce CG, et al. The cut-off limits of the $\mathrm{GH}$ response to $\mathrm{GH}$-releasing hormonearginine test related to body mass index. Eur J Endocrinol. 2005 Aug;153(2):257-64.

21 Aimaretti G, Boschetti M, Corneli G, Gasco V, Valle D, Borsotti M, et al. Normal age-dependent values of serum insulin growth factor-I: results from a healthy Italian population. J Endocrinol Invest. 2008 May;31(5): 445-9.

22 Zweig MH, Campbell G. Receiver-operating characteristic (ROC) plots: a fundamental evaluation tool in clinical medicine. Clin Chem. 1993 Apr;39(4):561-77.

23 Beardsell I, Bell S, Robinson S, Rumbold H. MCEM Part A, MCQs. Royal Society of Medicine Press; 2009.

24 Aimaretti G, Baffoni C, DiVito L, Bellone S, Grottoli S, Maccario M, et al. Comparisons among old and new provocative tests of $\mathrm{GH}$ secretion in 178 normal adults. Eur J Endocrinol. 2000 Apr;142(4):347-52.
25 Aimaretti G, Corneli G, Razzore P, Bellone S, Baffoni C, Arvat E, et al. Comparison between insulin-induced hypoglycemia and growth hormone (GH)-releasing hormone + arginine as provocative tests for the diagnosis of $\mathrm{GH}$ deficiency in adults. J Clin Endocrinol Metab. 1998 May;83(5):1615-8.

26 Aimaretti G, Bellone S, Baffoni C, Corneli G, Origlia C, Di Vito L, et al. Short Procedure of GHRH Plus Arginine Test in Clinical Practice. Kluwer Academic Publishers; 2001

27 Ghigo E, Goffi S, Nicolosi M, Arvat E, Valente F, Mazza E, et al. Growth hormone (GH) responsiveness to combined administration of arginine and $\mathrm{GH}$-releasing hormone does not vary with age in man. J Clin Endocrinol Metab. 1990 Dec;71(6):1481-5.

28 Darzy KH, Aimaretti G, Wieringa G, Gattamaneni HR, Ghigo E, Shalet SM. The usefulness of the combined growth hormone (GH)releasing hormone and arginine stimulation test in the diagnosis of radiation-induced GH deficiency is dependent on the post-irradiation time interval. J Clin Endocrinol Metab. 2003 Jan;88(1):95-102.

29 Ghigo E, Procopio M, Boffano GM, Arvat E, Valente F, Maccario M, et al. Arginine potentiates but does not restore the blunted growth hormone response to growth hormone-releasing hormone in obesity. Metabolism. 1992 May;41(5):560-3.

30 Procopio M, Maccario M, Grottoli S, Oleandri SE, Boffano GM, Camanni F, et al. Shortterm fasting in obesity fails to restore the blunted GH responsiveness to GH-releasing hormone alone or combined with arginine. Clin Endocrinol (Oxf). 1995 Dec;43(6):6659.

31 Maccario M, Valetto MR, Savio P, Aimaretti G, Baffoni C, Procopio M, et al. Maximal secretory capacity of somatotrope cells in obesity: comparison with GH deficiency. Int J Obes Relat Metab Disord. 1997 Jan;21(1):2732.

32 Maccario M, Gauna C, Procopio M, Di Vito L, Rossetto R, Oleandri SE, et al. Assessment of GH/IGF-I axis in obesity by evaluation of IGF-I levels and the GH response to GHRH+arginine test. J Endocrinol Invest. 1999 Jun;22(6):424-9.

33 Bonert VS, Elashoff JD, Barnett P, Melmed S. Body mass index determines evoked growth hormone $(\mathrm{GH})$ responsiveness in normal healthy male subjects: diagnostic caveat for adult GH deficiency. J Clin Endocrinol Metab. 2004 Jul;89(7):3397-401.
$34 \mathrm{Qu}$ XD, Gaw Gonzalo IT, Al Sayed MY, Cohan P, Christenson PD, Swerdloff RS, et al. Influence of body mass index and gender on growth hormone $(\mathrm{GH})$ responses to $\mathrm{GH}$-releasing hormone plus arginine and insulin tolerance tests. J Clin Endocrinol Metab. 2005 Mar;90(3):1563-9.

35 Ham JN, Ginsberg JP, Hendell CD, Moshang $\mathrm{T}$ Jr. Growth hormone releasing hormone plus arginine stimulation testing in young adults treated in childhood with cranio-spinal radiation therapy. Clin Endocrinol (Oxf). 2005 May;62(5):628-32.

36 Björk J, Link K, Erfurth EM. The utility of the growth hormone $(\mathrm{GH})$ releasing hormonearginine test for diagnosing GH deficiency in adults with childhood acute lymphoblastic leukemia treated with cranial irradiation. J Clin Endocrinol Metab. 2005 Nov;90(11): 6048-54.

37 Maghnie M, Cavigioli F, Tinelli C, Autelli M, Aricò M, Aimaretti G, et al. GHRH plus arginine in the diagnosis of acquired GH deficiency of childhood-onset. J Clin Endocrinol Metab. 2002 Jun;87(6):2740-4.

38 Bidlingmaier M, Freda PU. Measurement of human growth hormone by immunoassays: current status, unsolved problems and clinical consequences. Growth Horm IGF Res. $2010 \mathrm{Feb} ; 20(1): 19-25$

39 Tanaka T, Tachibana K, Shimatsu A, Katsumata N, Tsushima T, Hizuka N, et al. A nationwide attempt to standardize growth hormone assays. Horm Res. 2005;64 Suppl 2:6-11.

40 Clemmons DR. Consensus statement on the standardization and evaluation of growth hormone and insulin-like growth factor assays. Clin Chem. 2011 Apr;57(4):555-9.

41 Hoffman DM, O'Sullivan AJ, Baxter RC, Ho KK. Diagnosis of growth-hormone deficiency in adults. Lancet. 1994 Apr;343(8905):1064-8.

42 Biller BM, Samuels MH, Zagar A, Cook DM, Arafah BM, Bonert V, et al. Sensitivity and specificity of six tests for the diagnosis of adult GH deficiency. J Clin Endocrinol Metab. 2002 May;87(5):2067-79.

43 Whiting P, Rutjes AW, Reitsma JB, Glas AS, Bossuyt PM, Kleijnen J. Sources of variation and bias in studies of diagnostic accuracy: a systematic review. Ann Intern Med. 2004 Feb; 140(3):189-202.

44 Popovic V, Leal A, Micic D, Koppeschaar HP, Torres E, Paramo C, et al. GH-releasing hormone and GH-releasing peptide- 6 for diagnostic testing in $\mathrm{GH}$-deficient adults. Lancet. 2000 Sep;356(9236):1137-42.

45 Mahajan T, Lightman SL. A simple test for growth hormone deficiency in adults. J Clin Endocrinol Metab. 2000 Apr;85(4):1473-6. 